\title{
Dental calculus-a reservoir for detection of past SARS-CoV-2 infection
}

\author{
Federico Berton $^{1} \cdot$ Katia Rupel $^{1}$ (I) $\cdot$ Fiorella Florian $^{2} \cdot$ Matteo Biasotto $^{1} \cdot$ Alberto Pallavicini $^{2} \cdot$ Roberto Di Lenarda $^{1}$
}

Received: 14 March 2021 / Accepted: 20 May 2021 / Published online: 26 May 2021

(c) The Author(s), under exclusive licence to Springer-Verlag GmbH Germany, part of Springer Nature 2021

Keywords SARS-CoV-2 · Nasopharyngeal swabs · Dental calculus · COVID-19 · Serological testing

\section{Dear Sir/Madame,}

The SARS-CoV-2 outbreak left all the scientific and medical community with tremendous doubts and difficulties in managing both symptomatic and asymptomatic patients, especially in case of seronegativity [1,2]. In fact, in patients with mild symptoms, detectable IgG may not develop or progressively decrease [3]. The present letter aims to highlight an interesting finding, never mentioned before, that may help clinicians and epidemiologists in detecting previous SARS-CoV-2 infection. Learning from archaeological studies [4], we hypothesized that dental calculus, a mineralized oral plaque biofilm that contains biomolecules such as nucleic acids, could have preserved SARS-CoV-2 RNA after infection, regardless of the development and severity of symptoms.

Here, we present the results from a pilot study including five individuals with previous diagnosis of SARS-CoV-2 infection obtained by nasopharyngeal swab testing and subsequently healed within two months prior the study (POS), two individuals contact with a positive who developed mild symptoms and were negative at nasopharyngeal swab testing (SUSP), and five healthy individuals who denied contacts with positive subjects and had negative nasopharyngeal swabs (CTRL). All subjects underwent dental calculus sampling performed with sterile curettes. Samples were analyzed performing real-time reverse transcription polymerase chain reaction assays after nucleic acid extraction and amplification. The RdRp Institut Pasteur IP2 assay was used, as previous studies demonstrated its high sensitivity and specificity [5]. The results showed that SARS-CoV-2 RNA was detected in all POS subjects, in the two SUSP subjects, and one among CTRL subjects. Ct values are reported in Table 1. Furthermore, one of the POS subjects resulted negative at a serologic assay while the other four subjects developed specific IgG antibodies. Samples were further analyzed using the CDC N1 assay, confirming the presence of SARS-CoV-2 RNA in one POS subject. The presence of SARS-CoV-2 RNA was confirmed by genomic sequencing of the amplicons obtained with the RdRp Institut Pasteur IP2 assay. Sanger sequencing was run at Eurofins Genomics facilities.

Dental calculus has been previously analyzed, mostly for microbiome definition in archaeological human samples and serving as milieu for the preservation of genomic information. Similarly, dental calculus may be helpful to detect SARS-CoV-2 traces in previously infected and possibly also in asymptomatic or mild symptomatic patients that may not develop detectable antibodies, therefore lacking a method to confirm the occurred infection. Further studies are needed to define method's reliability, cost/effectiveness, and suitability for large-scale epidemiological studies or post-mortem analysis. Although our study has some limitations due to the small number of samples, we believe that its interest resides in the fact that it is the first describing the presence of SARS-CoV-2 RNA in dental calculus.

Katia Rupel

krupel@units.it

1 Department of Medicine, Surgery and Health Sciences, University of Trieste, Trieste, Italy

2 Department of Life Sciences, University of Trieste, Trieste, Italy 
Table 1 Characteristics of the subjects included in the pilot study

\begin{tabular}{lllllll}
\hline $\begin{array}{l}\text { Subject } \\
\text { number }\end{array}$ & Group & Symptoms & $\begin{array}{l}\text { Nasopharyn- } \\
\text { geal swab }\end{array}$ & Serological testing & Ct values (N1) & Ct values (IP2) \\
\hline 1 & SUSP & Mild & Negative & Negative & ND & 35,78 \\
2 & SUSP & Mild & Negative & Negative & ND & 35,71 \\
3 & POS & Present & Positive & Positive & ND & 37,28 \\
4 & POS & Present & Positive & Positive & ND & 41,07 \\
5 & POS & Present & Positive & Positive & ND & 33,37 \\
6 & POS & Present & Positive & Negative & 36,19 & 34,73 \\
7 & POS & Present & Positive & Positive & ND & 32,69 \\
8 & CTRL & Absent & Negative & Negative & ND & 37,25 \\
9 & CTRL & Absent & Negative & Negative & ND & ND \\
10 & CTRL & Absent & Negative & Negative & ND & ND \\
11 & CTRL & Absent & Negative & Negative & ND & ND \\
12 & CTRL & Absent & Negative & Negative & ND & ND \\
\hline
\end{tabular}

POS subjects with previous diagnosis of SARS-CoV-2 infection and healed at the time of sampling, SUSP individual contact with a subject with diagnosis of SARS-CoV-2 infection who developed mild symptoms, CTRL subjects who denied both contacts with positive subjects and symptoms, N1 primers and probes for PCR amplification of the N gene 28287-28358* (US Centers for Disease Control and Prevention CDC, USA), IP2 primers and probes for PCR amplification of the RdRp gene 12690-12797*, (Institute Pasteur, Paris), ND not detectable

Nucleotide numbering based on Wuhan-Hu-1 GenBank MN908997

\section{References}

1. Marklund E, Leach S, Axelsson H, Nyström K, Norder H, Bemark M, Angeletti D, Lundgren A, Nilsson S, Andersson LM, Yilmaz A, Lindh M, Liljeqvist JÅ, Gisslén M (2020) SerumIgG responses to SARS-CoV-2 after mild and severe COVID19 infection and analysis of IgG non-responders. PLoS ONE 15(10):e0241104. https://doi.org/10.1371/journal.pone.0241104

2. Oran DP, Topol EJ (2020) Prevalence of asymptomatic SARSCoV-2 infection: a narrative review. Ann Intern Med 173(5):362367. https://doi.org/10.7326/M20-3012

3. Petersen LR, Sami S, Vuong N, Pathela P, Weiss D, Morgenthau BM, Henseler RA, Daskalakis DC, Atas J, Patel A, Lukacs S, Mackey L, Grohskopf LA, Thornburg N, Akinbami LJ (2020) Lack of antibodies to SARS-CoV-2 in a large cohort of previously infected persons. Clin Infect Dis:ciaa1685. https://doi.org/ $10.1093 / \mathrm{cid} / \mathrm{ciaa} 1685$
4. Warinner C, Rodrigues JF, Vyas R, Trachsel C, Shved N, Grossmann J, Radini A, Hancock Y, Tito RY, Fiddyment S, Speller C, Hendy J, Charlton S, Luder HU, Salazar-García DC, Eppler E, Seiler R, Hansen LH, Castruita JA, Barkow-Oesterreicher S, Teoh KY, Kelstrup CD, Olsen JV, Nanni P, Kawai T, Willerslev E, von Mering C, Lewis CM Jr, Collins MJ, Gilbert MT, Rühli F, Cappellini E (2014) Pathogens and host immunity in the ancient human oral cavity. Nat Genet 46(4):336-344. https://doi.org/10. 1038/ng.2906

5. Etievant S, Bal A, Escuret V, Brengel-Pesce K, Bouscambert M, Cheynet V, Generenaz L, Oriol G, Destras G, Billaud G, Josset L, Frobert E, Morfin F, Gaymard A (2020) Performance assessment of SARS-CoV-2 PCR assays developed by WHO referral laboratories. J Clin Med 9(6):1871. https://doi.org/10.3390/jcm9061871

Publisher's note Springer Nature remains neutral with regard to jurisdictional claims in published maps and institutional affiliations. 